\title{
Effect of anthocyanin-rich Hungarian tart cherry extract on blood antioxidant status in $\mathrm{C} 57 \mathrm{BL} / 6 \mathrm{~J}$ mice
}

\author{
ANDREA NEMES ${ }^{1}$ - JUDIT R. HOMOKI ${ }^{1}$ - RITA KISS ${ }^{2}-$ \\ LÁSZLÓ STÜNDL ${ }^{1}$ - JUDIT REMENYIK ${ }^{1}$
}

1University of Debrecen, Institute of Food Technology, Debrecen, Hungary

${ }^{2}$ University of Debrecen, Faculty of Medicine, Institute of Pharmacology and Pharmacotherapy, Debrecen, Hungary

nemes.andrea@agr.unideb.hu

\begin{abstract}
Summary
In this study male C57BL/6J (B6) mice were used to determine the possible effects of our Hungarian tart cherry extract in a chronic obesity mouse model on antioxidant capacity.

The control group received standard mouse chow, the high fat control group was switched to high fat diet and tap water supplemented with $5 \%$ sucrose. The high fat+anthocyanin group received the high fat and sucrose diet, but dissolved in the drinking water they received anthocyanin-rich tart cherry extract. After six weeks, the antioxidant capacity and SOD activity were measured. Antioxidant capacity was decreased with a significant elevation of SOD activity. The tart cherry extract made a significant enhancement in antioxidant capacity and SOD activity. Our results show that chronic anthocyanin intake has a potential to enhance redox status associated with obesity.
\end{abstract}

Keywords: sour cherry, anthocyanins, obesity, type 2 diabetes, mouse

\section{Introduction}

Obesity is one of the most widespread metabolic diseases and also one of the biggest challenges for health care. Prevalence of obesity has increased threefold in the past decades, involving adults as well as children living not only in developed, but developing countries (Mirsa and Khurano, 2008).

The B6 mouse is an important model for understanidng the interplay between genetic background and environmental impacts (e.g. highfat/high-calorie diets) that predispose to the development of the metabolic syndrome. The B6 mouse is a particularly good model of the human metabolic syndrome because in case of high-fat diet it develops a syndrome of obesity, hyperinsulinemia, hyperglycemia, and hypertension (Surwit et al., 1988) as well as deteriorate plasma antioxidant status (Jeung et al., 2009). At the same time, the mouse remains lean and physically normal when receive low-fat chow. 
Sour cherries (Prunus cerasus) are very good source of natural antioxidants such as vitamins A, B1, B2, C, E, K, melatonin, anthocyanins and other phenolic compounds (Homoki et al., 2016). Anthocyanins are natural colorants belonging to the flavonoid family. They are widely distributed among flowers, fruits, and vegetables (Kong et al., 2003; Koponen et al., 2007; He and Giusti, 2010). Furthermore, anthocyanins have multiple biological roles for example they are able to reduce the production of reactive oxigen species (ROS), and in addition they can also scavenge excess ROS and protect antioxidant enzymes from inactivation (Gould et al., 2008). Moreover Wang et al. (1999) and Sarić et al. (2009) have demonstrated the anti-inflammatory properties of sour cherry anthocyanins (SCA), and that they increase the level of superoxide dismutase (SOD) in liver and blood, as well as Guo et al. (2008) proved SCA decrease the blood sugar level (and showed anti-obesity and antidiabetic effects). By historical results, we know Hungarian tart cherries containe for types of anthocyanin compounds: cyanidin-3-O-rutinoside, cyanidin-3-O-glucoside, malvidin-3,5-O-diglycoside and cyanidinglucosyl-rutinoside (Homoki et al., 2016) in extremely high concentrations.

\section{Material and methods}

Fruit samples

The Hungarian sour cherry „VN1" variety (selected from "Csengódi csokros") was selected for the experiment. The fruit samples originated from Research and Consulting Institute for Fruitgrowing, Újfehértó were collected between June and July of 2012, and were frozen immediately after picking, and were carried to the laboratory where they were kept in cold $\left(-20^{\circ} \mathrm{C}\right)$ dark store (Homoki et al., 2016).

\section{Preparation of Athocyanins}

The tart cherry sample was deseeded, homogenized with a Braun Multiquick mixer, and extracted with ethanol:water:acetic acid $=25: 24: 1$ solvent. The mixture was mixed using Magnetic stirrer MSH 300 (BIOSAN) for $1 \mathrm{~h}$, than filtered, centrifuged (SIGMA 2-16 SARTORIUS) for $5 \mathrm{~min}$ at $10000 \mathrm{rpm}$ and were further purified. For easier separation of anthocyanins, a simple fractionation of sour cherry extracts was performed using preconditioned Supelclean ENVI-18 SPE tubes (Kim et al., 2005). The tubes were conditioned with $5 \mathrm{ml} \mathrm{EtOH}$ then with $5 \mathrm{ml}$ $\mathrm{H}_{2} \mathrm{O}$ and finally $1 \mathrm{ml}$ of fruit sample was applied. The anthocyanins were eluted with ethanol containing $20 \%$ water. Solvent was evaporated at 40 ${ }^{\circ} \mathrm{C}$ with Heidolph rotary evaporator (Germany) (Homoki et al., 2016). 
Ethics

All experiments involving mice were conducted in accordance with the European Community guiding principles for care and use of experimental animals and the University of Debrecen Ethics Committee for Animal Research.

Thirty-five male C57BL/6J mice (obtained from Innovo Ltd, the local distributor of The Jackson Laboratory) were used throughout the study.

\section{Treatment of animals}

Male C57BL/6J mice were kept in polycarbone cages with controlled temperature $\left(22-24^{\circ} \mathrm{C}\right)$ and relative humidity level (50-70\%) under a $12 \mathrm{~h}$ light:dark cycle (lights off at $7 \mathrm{am}$ ). The animals were fed standard mouse chow (S8106- S011 SM R/M-Z+H; ssniff Spezialdiäten GmbH, Germany) and allowed free acces to tap water during the one-week acclimatization period.

Mice were randomly divided into three groups after acclimation. The diet of control group $(n=11)$ contained standard mouse chow and tap water ad libitum. The second group $(n=12)$ was switched to high fat diet (RM AFE 45\% FAT SY (P), Special Diets Services, United Kingdom) and tap water supplemented with $5 \%$ sucrose. The third group $(n=12)$ received high fat diet and sucrose as the second group did but dissolved in the drinking water they also received anthocyanin-rich tart cherry extract. The tart cherry extract and sucrose solutions were freshly prepared every day.

The experimental protocol lasted for six weeks. The water consumption was registered on a daily basis and the anthocyanin solution was prepared accordingly to maintain the daily dosage protocol $\left(60 \mathrm{mg} \mathrm{kg}^{-1}\right)$.

\section{Blood samples}

After the experimental period mice were sacrificed, blood samples were taken. $500 \mu \mathrm{l}$ whole, fresh blood was centrifuged for $10 \mathrm{~min}$ at $3000 \mathrm{rpm}$. Erythrocyte sediment ( $\max 500 \mu \mathrm{l})$ was re-suspended three times in 1.5 $\mathrm{ml}$ normal saline solution and centrifuged again for $5 \mathrm{~min}$ at $3000 \mathrm{rpm}$ each time. Supernatant was discarded. Lysis of the erythrocytes was achieved by adding $1 \mathrm{ml}$ cool water to the sediment and storing the sample at $4{ }^{\circ} \mathrm{C}$ in the dark for 15 minutes. Then it was centrifuged for 5 min at $4{ }^{\circ} \mathrm{C}$ at $3000 \mathrm{rpm}$. The upper phase was frozen and stored at $-70{ }^{\circ} \mathrm{C}$ for subsequent determinations. 
Determination of antioxidative capacity in water-solube substances (ACW) by photochemiluminescence method (PCL) using the Photochem instrument

The PCL method was carried out as described by Popov and Lewin (1994). In the PCL assay the photochemical generation of free radicals is combined with the sensitive detection by using chemiluminescence. The hydrophilic antioxidants of the plasma was measured in the Photochem with the ACW kit (Analytik Jena, Jena, Germany). $10 \mu$ prepared plasma, $1.5 \mathrm{ml}$ reagent 1 (buffer solution $\mathrm{pH}$ 10.5), $1 \mathrm{ml}$ reagent 2 (water) and 25 $\mu \mathrm{l}$ reagent 3 (photosensitizer) were mixed and measured. These are standardised conditions, so the results are comparable to other assays. The antioxidant potential was assayed by means of the lag phase.

Determination of the antioxidant capacityof the Enzyme SOD by PCL method using the Photochem instrument

The antioxidant capacity of the enzyme SOD were measured with the ACW kit (Analytik Jena, Jena, Germany). $10 \mu \mathrm{l}$ prepared red blood cell (RBC), $1.5 \mathrm{ml}$ reagent $1,1 \mathrm{ml}$ reagent 2 and $25 \mu \mathrm{l}$ reagent 3 (photosensitizer) were mixed and measured. SOD-enzyme (Superoxide Dismutase, Sigma Aldrich, Germany) was used as standard (Popov and Lewin, 1999).

\section{Statistics}

Data were presented as mean $\pm \mathrm{SEM}$. All data were analyzed with oneway analysis of variance (ANOVA) followed by a modified t-test for repeated measures according to Tukey's method.

\section{Results}

Effect of anthocyanin treatment on ACW

Table 1 represents the effect of anthocyanin treatment on plasma levels of ACW. In case of mouse (1-12), the values are mean of the three replicates $\pm S D$. The average data show the mean of the all values in each group.

As shown, ACW concentrations were reduced by half due to high fat diet. This difference is significant between the control and high fat control group. Anthocyanin treatment could effectively prevent the decrease, furthermore, high fat+anthocyanin mice even showed a slight, but significant increase in ACW levels compared to healthy controls.

Effect of anthocyanin treatment on SOD

The values the SOD enzyme of RBC was presented in Table 2. Because of higt fat diet and sucrose, the levels of SOD increased significantly in high fat control group by the end of the experimental period. It is an increase 
of 12 percent between the control and the high fat control groups. On the basis of results, anthocyanin treatment effectively prevented the differences seen in high fat controls.

Table 1. The effect of anthocyanin treatment on plasma levels of ACW

\begin{tabular}{lrcc}
\hline Number of mouse & Control & High fat control & High fat + anthocyanin \\
\hline 1 & $10.21 \pm 0.16$ & $4.83 \pm 0.08$ & $10.17 \pm 0.35$ \\
2 & $9.87 \pm 0.02$ & $5.10 \pm 0.36$ & $10.38 \pm 0.23$ \\
3 & $9.36 \pm 0.40$ & $4.86 \pm 0.12$ & $10.63 \pm 0.34$ \\
4 & $9.02 \pm 0.13$ & $5.00 \pm 0.16$ & $10.14 \pm 0.36$ \\
5 & $9.42 \pm 0.07$ & $5.35 \pm 0.14$ & $11.78 \pm 0.34$ \\
6 & $9.93 \pm 0.21$ & $4.59 \pm 0.11$ & $10.84 \pm 0.29$ \\
7 & $9.42 \pm 0.18$ & $5.17 \pm 0.03$ & $10.20 \pm 0.20$ \\
8 & $9.94 \pm 0.08$ & $4.73 \pm 0.06$ & $11.32 \pm 0.16$ \\
9 & $10.21 \pm 0.06$ & $4.74 \pm 0.08$ & $11.65 \pm 0.19$ \\
10 & $9.99 \pm 0.09$ & $4.25 \pm 0.04$ & $11.24 \pm 0.33$ \\
11 & $9.53 \pm 0.09$ & $4.50 \pm 0.12$ & $11.38 \pm 0.50$ \\
12 & - & $4.80 \pm 0.05$ & $11.79 \pm 0.16$ \\
Average & $9.72 \pm 0.19$ & $4.82 \pm 0.15^{*}$ & $10.96 \pm 0.32^{*} \#$ \\
\hline Note: the & ind &
\end{tabular}

Note: the * indicates significant difference $(\mathrm{p}<0.05)$ from the control group. The \# indicates significant difference $(\mathrm{p}<0.05)$ between the high fat control and the high fat+anthocyanin groups.

Table 2. The effect of anthocyanin treatment on plasma levels of SOD

\begin{tabular}{lccc}
\hline Number of mouse & Control & High fat control & High fat + anthocyanin \\
\hline 1 & $168.23 \pm 1.55$ & $192.78 \pm 1.46$ & $178.26 \pm 2.12$ \\
2 & $172.61 \pm 1.12$ & $184.16 \pm 1.86$ & $168.92 \pm 1.97$ \\
3 & $167.93 \pm 1.13$ & $175.69 \pm 2.03$ & $168.20 \pm 2.66$ \\
4 & $167.26 \pm 1.05$ & $190.84 \pm 0.89$ & $168.64 \pm 2.03$ \\
5 & $167.33 \pm 1.44$ & $195.17 \pm 0.67$ & $164.59 \pm 1.37$ \\
6 & $172.53 \pm 1.81$ & $187.77 \pm 0.72$ & $172.69 \pm 0.24$ \\
7 & $167.11 \pm 1.21$ & $190.44 \pm 1.47$ & $169.44 \pm 2.62$ \\
8 & $167.45 \pm 2.41$ & $195.88 \pm 1.03$ & $173.08 \pm 0.93$ \\
9 & $163.42 \pm 0.72$ & $183.05 \pm 1.70$ & $171.11 \pm 2.39$ \\
10 & $172.38 \pm 1.94$ & $191.31 \pm 1.63$ & $166.35 \pm 1.36$ \\
11 & $172.28 \pm 1.83$ & $191.63 \pm 0.42$ & $166.63 \pm 1.01$ \\
12 & - & $193.53 \pm 1.13$ & $167.08 \pm 0.71$ \\
Average & $168.96 \pm 1.52$ & $189.35 \pm 2.92^{*}$ & $169.58 \pm 1.87 \#$ \\
\hline Note: the & indicates
\end{tabular}

Note: the * indicates significant difference $(\mathrm{p}<0.05)$ from the control group. The \# indicates significant difference $(\mathrm{p}<0.05)$ between the high fat control and the high fat+anthocyanin groups.

\section{Conclusion}

In our in vivo experiment the combination of high fat diet and drinking water supplemented with sucrose significantly decreased levels of ACW in the six week experimental period, and increased levels of SOD. 
However, anthocyanin treatment in obese mice effectively prevented the differences seen in high fat controls.

In sum, our group demonstrated that anthocyanin intake has a potential to enhance redox status.

\section{References}

Chavez-Santoscoy, R. A.-Gutierrez-Uribe, J. A.-Serna-Saldivar, S. O. (2009): Phenolic composition, antioxidant capacity and in vitro cancer cell cytotoxicity of nine prickly pear (Opuntia spp.) juices. Plant Foods for Human Nutrition. 64. 2: 146-152.

Espin, J. C.-Soler-Rivas, C.-Wichers, H. J. (2000): Characterization of the total free radical scavenging capacity of vegetable oils and oil fractions using 2,2diphenyl-1-picrylhydrazyl radical. Journal of Agricultural and Food Chemistry. 48. 3: 648-656.

Gould, K.-Davies, K.-Winefield, C. (2008): Anthocyanins: Biosynthesis, Functions, and Applocations. Springer Science \& Business Media LLC. New York.

Guo, H.-Ling, W.-Wang, Q.-Liu, C.-Hu, Y.-Xia, M. (2008): Cyanidin 3-glucoside protects 3T3-L1 adipocytes against $\mathrm{H}_{2} \mathrm{O}_{2}$ - or TNF-a- induced insulin resistance by inhibiting c-Jun NH2-terminal kinase activation. Biochemical Pharmacology. 75. 6: 1393-1401.

He, J.-Giusti, M. M. (2010): Anthocyanins: natural colorants with healthpromoting properties. Annual Review of Food Science and Technology. 1: 163-187.

Homoki, J. R.-Nemes, A.-Fazekas, E.-Gyémánt, G.-Balogh, P.-Gál, F.-Al-Asri, J.Mortier, J.-Wolber, G.-Babinszky, L.-Remenyik, J. (2016): Anthocyanin composition, antioxidant efficiency, and a-amylase inhibitor activity of different Hungarian sour cherry varieties (Prunus cerasus L.). Food Chemistry. 194: 222-229.

Jeung, H. L.-Chan, W. S.-Mi, Y. K.-Min, H. K.-Hye, R. K.-Eun, S. K.-Sena, K.-Mee, R. K. (2009): Red beet (Beta vulgaris L.) leaf supplementation improves antioxidant status in C57BL/6J mice fed high fat high cholesterol diet. Nutrition Research and Practice. 3. 2: 114-121.

Kim, D.-Heo, H. J.-Yang, H. S.-Lee, C. Y. (2005): Sweet and sour cherry phenolics and their protective effects on neuronal cells. Journal of Agricultural and Food Chemistry. 53. 26: 9921-9927.

Kong, J. M.-Chia, L. S.-Goh, N. K.-Chia, T. F.-Brouillard, R. (2003): Analysis and biological activities of anthocyanins. Phytochemistry. 64. 5: 923-933.

Koponen, J. M.-Happonen, A. M.-Mattila, P. H.-Torronen, A. R. (2007): Contents of anthocyanins and ellagitannins in selected foods consumed in Finland. Journal of Agricultural and Food Chemistry. 55. 4: 1612-1619.

Mazza, G.-Miniati, E. (1993): Anthocyanins in fruits, vegetables and grain. CRC Press. Florida.

Mirsa, A.-Khurano, L. (2008): Obesity and the metabolic syndrome in developing countries. The Journal of Clinical Endocrinology and Metabolism. 93. 1: S9-30. 
Motohashi, N.-Sakagami, H. (2009): Anthocyanins as Functional Food Colors. [In: de Hoop, H.-de Swart, P. (eds.) Linguistics. Bioactive Heterocycles VII.] Springer-Verlag. Berlin. 1-40.

Popov, I. N.-Lewin, G. (1994): Photochemiluminescent detection of antiradical activity. 2. Testing nonenzymic water-soluble antioxidants. Free Radical Biology and Medicine. 17. 3: 267-271.

Popov, I. N.-Lewin, G. (1999): Antioxidative Homeostasis: Characterisation by Means of Chemoluminescent Technique. Methods in Enzymology. 300: 437456.

Reddy, M. K.-Alexander-Lindo, R. L.-Nair, M. G. (2005): Relative inhibition of lipid peroxidation, cyclooxygenase enzymes, and human tumor cell proliferation by natural food colors. Journal of Agricultural and Food Chemistry. 53. 23: 9268-9273.

Sarić, A.-Sobocanec, S.-Balog, T.-Kusić, B.-Sverko, V.-Dragović-Uzelac, V.-Levaj, B.Cosić, Z.-Macak Safranko, Z.-Marotti, T. (2009): Improved antioxidant and antiinflammatory potential in mice consuming sour cherry juice (Prunus cerasus cv. Maraska). Plant Foods for Human Nutrition. 64. 4: 231-237.

Surwit, R. S.-Kuhn, C. M.-Cochrane, C.-McCubbin, J. A.-Feinglos, M. N. (1998): Diet-induced type II diabetes in C57BL/6J mice. Diabetes. 37: 1163-1167.

Tsuda, T.-Shiga, K.-Ohshima, K.-Kawakishi, S.-Osawa, T. (1996): Inhibition of lipid peroxidation and the active oxygen radical scavenging effect of anthocyanin pigments isolated fro Phaseolus vulgaris L. Biochemical Pharmacology. 52. 7: 1033-1039.

Wang, H.-Nair, M. G.-Strasburg, G. M. (1999): Antioxidant and antiinflammatory activities of anthocyanins and their aglycon, cyanidin, from tart cherries. Journal of Natural Products. 62. 2: 294-296.

Williams, G.-Frühbeck, G. (2008): Obesity: Science to Practice. First Edition. WileyBlackwell. Oxford. 